\title{
AnOMalous Origin of LeFt Circumflex ARTERY From the Right Coronary Artery
}

\author{
Sivakumar Srinivasan, MD, and Sandeep Anreddy, MD
}

\section{Case Presentation}

A 56 year-old male with past medical history significant for diabetes and obesity presented for an elective diagnostic cardiac catheterization. The catheterization films are shown below (Figures 1 and 2).

\section{Discussion}

Adult coronary anomalies are not very common and are usually casual findings of diagnostic angiographic studies. The incidence of these anomalies has been reported to be between 0.29 $1.34 \%$. Although coronary artery anomalies are far less common than acquired coronary artery disease, their impact on premature cardiac

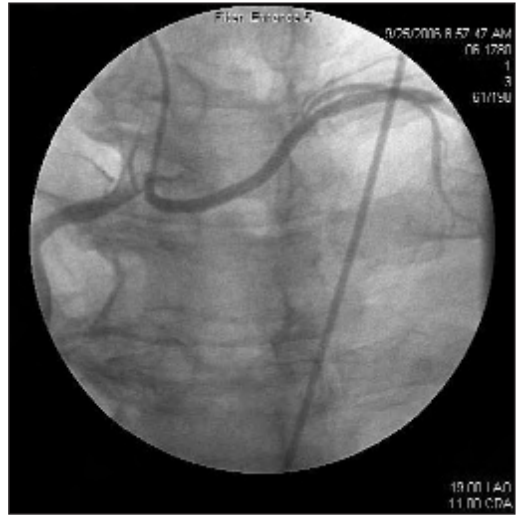

Figure 1.

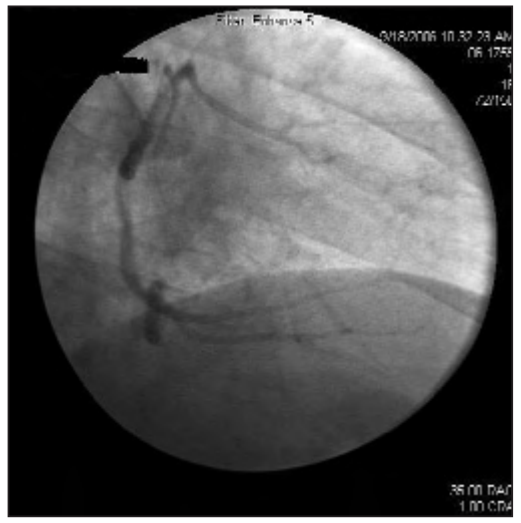

Figure 2. morbidity and mortality among young adults is not trivial. In a study of 126 nontraumatic sudden deaths in young adults, cardiac abnormality was found in 64 cases $(51 \%)$, with coronary artery abnormalities being the most common cardiac abnormality(61\%).

Coronary artery anomalies can be classified as hemodynamically significant or insignificant. Hemodynamically significant anomalies of the coronary arteries are characterized by abnormalities of myocardial perfusion, which lead to an increased risk of myocardial ischemia or sudden death. These anomalies include an anomalous origin of either the LCA or the RCA from the pulmonary artery, an anomalous course between the pulmonary artery and the aorta (interarterial) of either the RCA arising from the left sinus of valsalva or the LCA arising from the right sinus of valsalva, occasional myocardial bridging, and congenital coronary artery fisula.

Anomalous origin of the coronary artery from the pulmonary artery (ALCAPA) is one of the most serious congenital coronary artery anomalies. It has an estimated prevalence of one in 300,000 live births. Most affected patients show symptoms in infancy and early childhood. Approximately $90 \%$ of untreated infants die in the 1 st year of life, and only a few patients survive to adulthood. In the most common form of this disease, the LCA arises from the pulmonary artery and the RCA arises normally from the aorta (Bland-White-Garland syndrome).

The four recognized patterns of an anomalous origin of a coronary artery from the opposite or noncoronary sinus are (a) the RCA arising from the left coronary sinus, (b) the LCA arising from the right coronary sinus, (c) the LCx or LAD artery arising from the right coronary sinus, and (d) the LCA or RCA (or a branch of either artery) arising from the noncoronary sinus. A coronary artery arising from the opposite or noncoronary sinus can take any of four common courses, depending on the anatomic relationship of the anomalous vessel to the aorta and the pulmonary trunk: (a)interarterial (ie, between the aorta and the pulmonary artery),

(b) retroaortic, (c) prepulmonic, or (d) septal (subpulmonic). It is of great clinical importance which course is taken, an interarterial course carries a high risk for sudden cardiac death. The most common course of an anomalous RCA arising from the left sinus of Valsalva is interarterial; this variant can be associated with sudden cardiac death in up to $30 \%$ of patients. It has been postulated that, when dilation of the aorta occurs during exercise, the anomalous slit-like ostium for the RCA in the left sinus becomes narrower, possibly limiting coronary blood flow and resulting in myocardial infarction. The LCA arises from the right sinus of valsalva in $0.09 \%-0.11 \%$ of patients who undergo angiography, an interarterial course may be seen in up to $75 \%$ of patients with this anomaly.

Anomalous origin of the Left circumflex artery (LCx) from the proximal right coronary artery (RCA) is one of the most common forms of coronary artery anomalies. It has been reported in $0.17 \%-0.4 \%$ of patients undergoing coronary angiography. In almost all cases where the LCx arises from the proximal RCA the course is retroaortic. Results from the coronary artery surgery study showed that anomalous circumflex coronary arteries had a significant greater degree of stenosis when compared to nonanomalous arteries $(\mathrm{p}=0.02)$ though there was no significant difference in survival by location or degree of stenosis in the anomalous artery at 7 years.

\section{References}

1. Wilkins CE, Betancourt B, Mathur VS, et al. Coronary artery anomalies: A review of more than 10,000 patients from the Clayton Cardiovascular Laboratories. Tex Heart Inst J 1988; 15:166-173.

2. Yamanaka O, Hobbs RE. Coronary artery anomalies in 126,595 patients undergoing coronary arteriography. Cathet Cardiovascular Diagn 1990;21:28-40.

3. Eckart RE, Scoville SL, Campbell CL, et al. Sudden death in young adults: a 25-year review of autopsies in military recruits. Ann Intern Med 2004; 141:829-834.

4. Reagan K, Boxt LM, Katz J. Introduction to coronary arteriography. Radiol Clin North Am 1994; 32:419-433.

5. So Yeon Kim, Joon Beom Seo et al. Coronary Artery Anomalies: Classification and ECG-gated Multi-Detector Row CT Findings with Angiographic Correlation. RadioGraphics, Mar 2006; 26: 317 - 333 